\title{
Letter to the Editor: Enhanced Recovery After Surgery for Low- and Middle-Income Countries
}

\author{
Felipe Quezada ${ }^{1,2}$
}

Published online: 23 March 2018

(c) Société Internationale de Chirurgie 2018

\section{Dear Editor,}

I read with interest the editorial perspective of McQueen et al. regarding Enhanced Recovery After Surgery (ERAS) protocols for low- and middle-income countries [1]. The authors make an excellent description and offer possible solutions to the difficult task to initiate an ERAS programs in this setting, conditions that have been described as major barriers by healthcare providers before the program has even started [2]. Nevertheless, one important issue to add in this discussion is the adoption of ERAS programs in countries considered part of the high-income category but also with high-demanding health systems and limited available resources, as is the case in Chile. Currently, Chile has a mixed public and private healthcare system, where $73 \%$ of the national population is part of the public system (including the most vulnerable social and economic group) [3], making the development of healthcare policies to optimize our limited resources vital.

In that line of work, our group implemented since 2016 an ERAS program for colorectal surgery in one of the largest public hospitals in Chile, achieving in the first year of its implementation, a 2-day decrease in the median hospital stay for elective colorectal surgical procedures, without increased morbidity or hospital readmissions.

Currently we have enrolled more than 120 patients, and we are seeking to expand the inclusion criteria and

Felipe Quezada

ffquezad@uc.cl

1 Colorectal Surgery Unit, Department of Surgery, Complejo Asistencial Doctor Sótero del Río, Avenida Concha y Toro 3459, 8207257 Santiago, Región Metropolitana, Chile

2 Colorectal Surgery Unit, Department of Digestive Surgery, Pontificia Universidad Católica de Chile, Diagonal Paraguay 362, 8330077 Santiago, Región Metropolitana, Chile interventions and evaluate the cost burden on the public healthcare system, focusing on the rational use of economic resources as is described elsewhere [4].

The implementation of an ERAS program in this setting is also a demanding endeavor and specific strategies should also be developed for this particular setting. Successful ERAS programs require a series of cognitive and social processes such as strong leadership and constructive relationships within the healthcare team, aiming toward the development of patient-centered care policies, despite the presence of limited resources.

Compliance with ethical standards

Conflict of interest The author declares he has no conflict of interest.

\section{References}

1. McQueen K, Oodit R, Derbew M et al (2018) Enhanced recovery after surgery for low-and middle-income countries. World J Surg. https://doi.org/10.1007/s00268-018-4481-5

2. Alawadi Z, Leal I, Phatak U et al (2016) Facilitators and barriers of implementing enhanced recovery in colorectal surgery at a safety net hospital: a provider and patient perspective. Surgery 159:700-712

3. Cuenta pública participativa, Fonasa 2015 (2015) https://participa cionciudadana.fonasa.cl/portal_fonasa/site/artic/20160314/asoc file/20160314171054/cuenta_publica_2016_version_final_1.pdf. Accessed 21 Mar 2018

4. Rossi G, Vaccarezza H, Alvarez A (2015) Two-day hospital stay after laparoscopic colorectal surgery, is enhanced recovery program a health care system specific issue? Reply. World J Surg 39:1331-1332. https://doi.org/10.1007/s00268-015-3000-1 